\title{
Improved Candidate Drug Mining for Alzheimer's Disease
}

\author{
Yu-Huei Cheng, ${ }^{1}$ Li-Yeh Chuang, ${ }^{2}$ Hsueh-Wei Chang, ${ }^{3,4,5,6}$ and Cheng-Hong Yang ${ }^{7}$ \\ ${ }^{1}$ Department of Digital Content Design and Management, Toko University, Chiayi 613, Taiwan \\ ${ }^{2}$ Department of Chemical Engineering, Institute of Biotechnology and Chemical Engineering, I-Shou University, \\ Kaohsiung 84001, Taiwan \\ ${ }^{3}$ Department of Biomedical Science and Environmental Biology, Kaohsiung Medical University, Kaohsiung 80708, Taiwan \\ ${ }^{4}$ Translational Research Center, Kaohsiung Medical University Hospital, Kaohsiung Medical University, Kaohsiung 80708, Taiwan \\ ${ }^{5}$ Cancer Center, Kaohsiung Medical University Hospital, Kaohsiung Medical University, Kaohsiung 80708, Taiwan \\ ${ }^{6}$ Institute of Medical Science and Technology, National Sun Yat-Sen University, Kaohsiung 80424, Taiwan \\ ${ }^{7}$ Department of Electronic Engineering, National Kaohsiung University of Applied Sciences, Kaohsiung 80778, Taiwan
}

Correspondence should be addressed to Hsueh-Wei Chang; changhw2007@gmail.com and

Cheng-Hong Yang; chyang@cc.kuas.edu.tw

Received 25 December 2013; Accepted 19 January 2014; Published 27 February 2014

Academic Editor: Wei Chiao Chang

Copyright (C) 2014 Yu-Huei Cheng et al. This is an open access article distributed under the Creative Commons Attribution License, which permits unrestricted use, distribution, and reproduction in any medium, provided the original work is properly cited.

\begin{abstract}
Alzheimer's disease (AD) is the main cause of dementia for older people. Although several antidementia drugs such as donepezil, rivastigmine, galantamine, and memantine have been developed, the effectiveness of AD drug therapy is still far from satisfactory. Recently, the single nucleotide polymorphisms (SNPs) have been chosen as one of the personalized medicine markers. Many pharmacogenomics databases have been developed to provide comprehensive information by associating SNPs with drug responses, disease incidence, and genes that are critical in choosing personalized therapy. However, we found that some information from different sets of pharmacogenomics databases is not sufficient and this may limit the potential functions for pharmacogenomics. To address this problem, we used approximate string matching method and data mining approach to improve the searching of pharmacogenomics database. After computation, we can successfully identify more genes linked to AD and $\mathrm{AD}$-related drugs than previous online searching. These improvements may help to improve the pharmacogenomics of AD for personalized medicine.
\end{abstract}

\section{Introduction}

Alzheimer's disease (AD), the most common form of dementia, was first reported in 1906 [1]. In 2006, there were about 26.6 million $\mathrm{AD}$ patients worldwide and it was also common in southern Taiwan [2]. Although AD has been identified for a long time, most research progress was made in the recent 30 years [3]. However, no definitive cure is available for this disease and eventually it leads to death. Therefore, the drug discovery for Alzheimer's disease remains challenging.

Single nucleotide polymorphisms (SNPs) are the most common variation in human genomes [4]. The importance of SNPs has been reviewed in genome-wide association studies for its association with disease susceptibility and drug metabolism $[5,6]$. About $60-90 \%$ of the individual variation of drug response depends on pharmacogenomic factors. Therefore, SNP genotyping for candidate genes, pharmacological research, and drug discovery may play an increasingly important role in AD treatment. Meanwhile, increasing amounts of related information require the assistance of bioinformatics to construct the suitable databases and web servers.

Recently, PharmGKB (the Pharmacogenetics and Pharmacogenomics Knowledge Base) has been constructed to provide a comprehensive database for pharmacogenomic studies [7]. PharmGKB provides the pharmacogenetics research network in terms of SNP discovery and drug responses [8] with the fully curated knowledge for drug pathways, drug-related genes, and relationships among genes, drugs, and diseases. However, some information of different functions of PharmGKB is insufficient to allow convenient crosstalking between each other. 


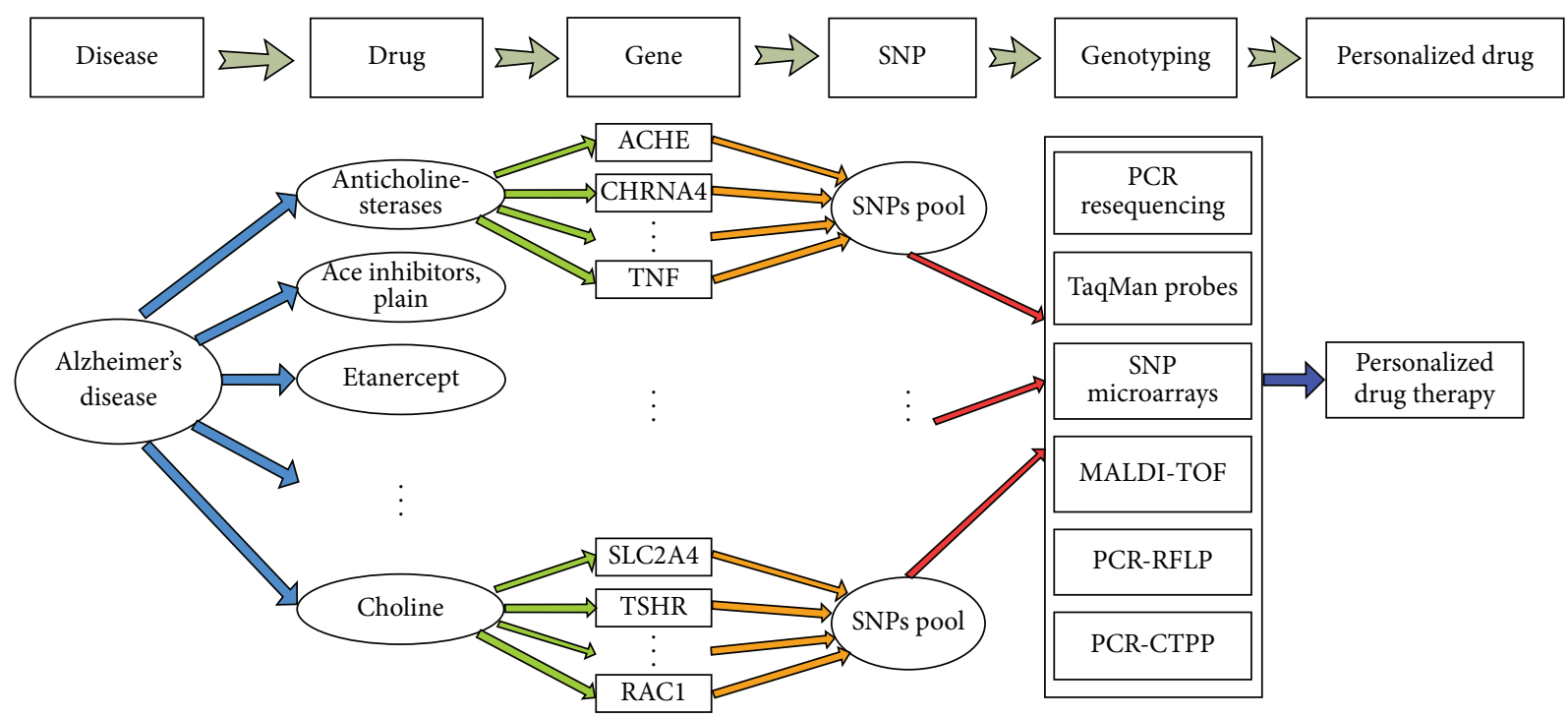

FIgURE 1: The flowchart for PharmGKB-based pharmacogenomics of AD in this study.

To solve this problem, we propose data mining method to improve the searching of pharmacogenomics of AD based on the download dataset of the PharmGKB resource.

\section{Materials and Methods}

The flowchart for pharmacogenomics in $\mathrm{AD}$ for personalized drug studies is shown in Figure 1. First of all, the AD-related drugs and genes are retrieved from PharmGKB download data using approximate string matching method and data mining approach. The genes associated with $\mathrm{AD}$ and the genes associated with a single Alzheimer's drug are identified and compared with the online searching of PharmGKB. Then, numerous SNPs of genes associated with AD are identified. Through some SNP genotyping tools or assays, the association studies to $\mathrm{AD}$-related drugs may be evaluated. Finally, the relevant information may be helpful for the personalized drug research.

2.1. AD-Related Drugs Using Approximate String Matching Based on PharmGKB Download Data. In order to study the pharmacogenomics of $\mathrm{AD}$, we downloaded the known PharmGKB (the Pharmacogenetics and Pharmacogenomics Knowledge Base) (http://www.pharmgkb.org/downloads/) $[9,10]$ as source by the approximate string matching method [11] to find out all AD-related drug classes. The meaningful keywords associated with "Alzheimer's disease" are shown in Table 1. Then, these found drug classes are used to find out associated genes by data mining approach. The description of the approximate string matching method for all AD-related drug classes gives a pattern string $P=p_{1} p_{2} p_{3} \cdots p_{m}$, that is, the meaningful keywords associated with "Alzheimer's disease" and a text string $T=t_{1} t_{2} t_{3} \cdots t_{n}$, that is, the description for drug and disease retrieved from PharmGKB. Find a substring $T_{i, j}=t_{i} t_{i+1} t_{i+2} \cdots t_{j}$ in $T$ that has the smallest edit distance [12] to the pattern $P$. The pseudocode for the edit distance is shown in Algorithm 1.

2.2. Data Mining Method for PharmGKB Download Data. In this study, we used a priori algorithm [13] for frequent item set mining and association rule learning over PharmGKB. The pseudocode for the a priori algorithm for data mining in PharmGKB is shown in Algorithm 2. At first, a priori algorithm has to find out the frequent gene in drug class for "Alzheimer's disease." A set of genes can be mined from each drug class. A priori algorithm is a "bottom up" approach, where frequent gene subsets are extended one item at a time (i.e., candidate generation) and groups of candidates are tested against the data. This algorithm is terminated when no further successful extensions are found.

2.3. SNP Searching for Genes Using the NCBI dbSNP. Every gene contains numerous SNPs. In order to find out SNPs of single gene for Alzheimer's pharmacogenomics, NCBI dbSNP (http://www.ncbi.nlm.nih.gov/snp) is used to search in the study.

\section{Results and Discussion}

3.1. AD Information Based on PharmGKB Search. In PharmGKB online searching, the SNP variants, related genes, and drugs for $\mathrm{AD}$ are able to be retrieved. For example, the SNP information such as rs2066853 and rs6313 is provided (Figure 2). As shown in Figure 3, the AD-related genes such as ADRB1, AHR, HTR2A, MTHFR, and PTGS2 are identified and the related drugs such as olanzapine and risperidone are searched. This information may assist the researchers to study the pharmacogenomics of AD. Unfortunately, this 
TABLE 1: The meaningful keywords associated with "Alzheimer's disease" are retrieved from PharmGKB and they are applied to discover the drug classes*.

\begin{tabular}{|c|c|}
\hline ID & Keywords \\
\hline 1 & $\mathrm{AD}$ \\
\hline 2 & Alzheimer's disease \\
\hline 3 & AD_Alzheimer's disease \\
\hline 4 & Acute Confusional Senile Dementia \\
\hline 5 & Alzheimer Dementia, Presenile \\
\hline 6 & Alzheimer Disease, Early Onset \\
\hline 7 & Alzheimer Disease, Late Onset \\
\hline 8 & Alzheimer Type Dementia \\
\hline 9 & Alzheimer Type Senile Dementia \\
\hline 10 & Alzheimer's Disease, Focal Onset \\
\hline 11 & Alzheimer's disease, NOS \\
\hline 12 & Dementia, Alzheimer Type \\
\hline 13 & Dementia, Presenile \\
\hline 14 & Dementia, Presenile Alzheimer \\
\hline 15 & Dementia, Primary Senile Degenerative \\
\hline 16 & Dementia, Senile \\
\hline 17 & Dementias, Presenile \\
\hline 18 & Dementias, Senile \\
\hline 19 & Disease, Alzheimer \\
\hline 20 & Disease, Alzheimer's \\
\hline 21 & Early Onset Alzheimer Disease \\
\hline 22 & Focal Onset Alzheimer's Disease \\
\hline 23 & Late Onset Alzheimer Disease \\
\hline 24 & Presenile Alzheimer Dementia \\
\hline 25 & Presenile Dementia \\
\hline 26 & Presenile Dementias \\
\hline 27 & Primary Senile Degerative Dementia \\
\hline 28 & Senile Dementia \\
\hline 29 & Senile Dementia, Acute Confusional \\
\hline 30 & Senile Dementia, Alzheimer Type \\
\hline 31 & Senile Dementias \\
\hline 32 & MeSH: D000544 (Alzheimer Disease) \\
\hline 33 & MedDRA: 10001896 (Alzheimer’s disease) \\
\hline 34 & NDFRT: N0000000363 (Alzheimer Disease [Disease/Finding]) \\
\hline 35 & SnoMedCT: 26929004 (Alzheimer's disease) \\
\hline 36 & UMLS: C0002395 (C0002395) \\
\hline
\end{tabular}

${ }^{*}$ Drug class is one of the functions listed in the ParamGKB download data.

PharmGKB online searching just provides limited information and it insufficiently copes with the complexity of the drug researches for Alzheimer's personalized medicine.

3.2. PharmGKB-Based Data Mining of AD Information of Drug Classes or Gene Symbols. In current study, our proposed method is used to perform data mining for PharmGKB download data in terms of the keyword "Alzheimer's disease." As shown in Table 2, 22 kinds of AD-related drug classes are identified from "drug classes" of PharmGKB. Their corresponding PharmGKB accession ID, PubMed PMID, and the number of genes that are associated with $\mathrm{AD}$-related drug 


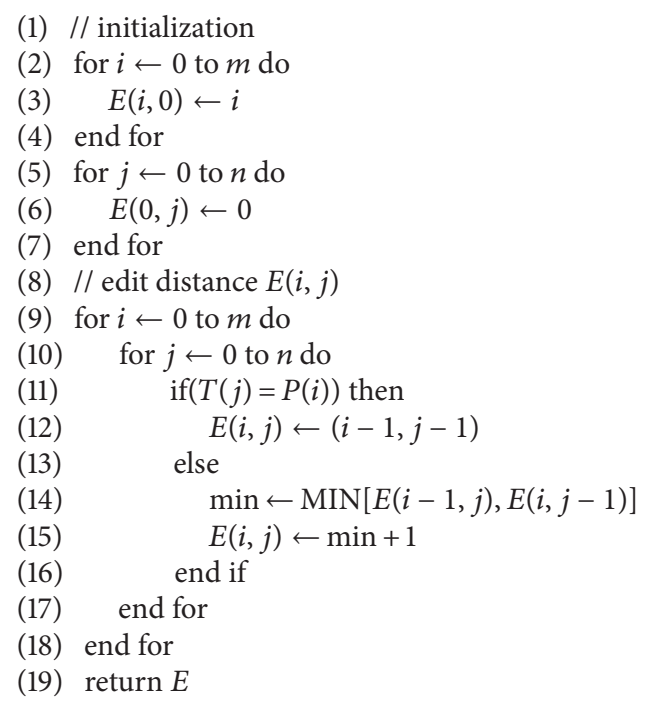

Algorithm 1: Pseudocode for the edit distance used for approximate string matching.

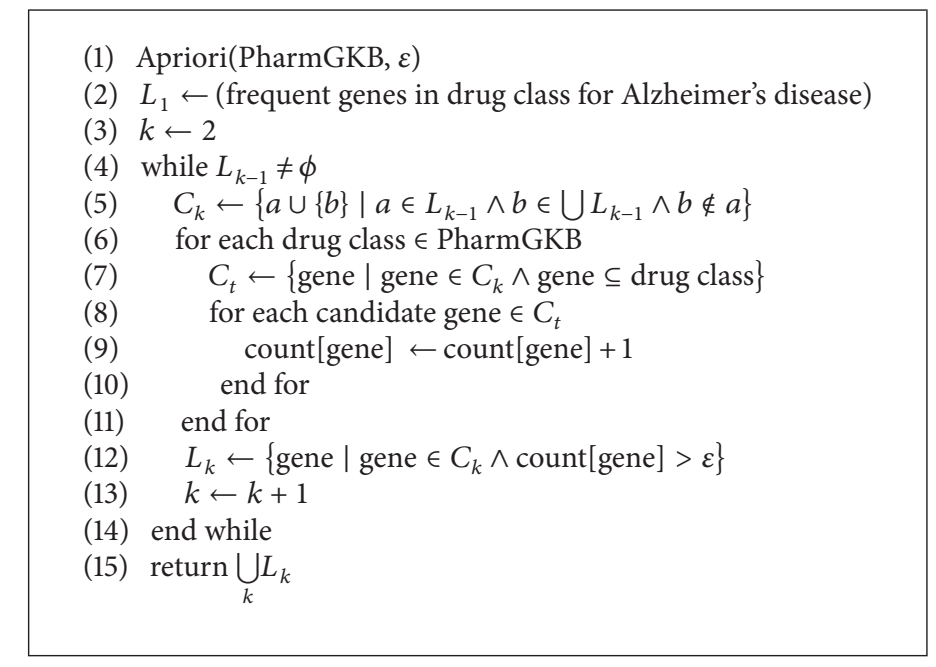

Algorithm 2: Pseudocode for a priori algorithm for the data mining in PharmGKB, where $\varepsilon$ is a support threshold, $L$ is the frequent gene subsets that satisfy the support threshold, $k$ is the number of current iterations, and $C$ is the candidate set, and count[gene] accesses a field of the data structure that represents gene candidate set.

classes are also presented. In total, 495 genes are identified for AD information of drug classes (see Supplementary file 1: gene information includes PharmGKB Accession Id, gene symbol, and publications are providing in different classes; it is available online at http://dx.doi.org/10.1155/2014/897653). Alternatively, 99 genes associated with $\mathrm{AD}$ are identified from "gene symbols" of PharmGKB in terms of the keyword "Alzheimer's disease." These results suggest that the same keyword, for example, Alzheimer's disease, may identify different numbers of $\mathrm{AD}$-associated genes between "drug classes" or "gene symbols" of PharmGKB.

After detailed examination, 67 genes in the gene symbols searching (bold fonts of gene names as shown in Table 3 ) are absent from the genes in the drug class searching (Table 2).
Furthermore, genes corresponding to the drug "memantine" listed in Table 2 (drug classes) are not found in Table 3 (gene symbols). Therefore, some current drugs have identified a small number of AD-related genes in the drug class searching; however, the remaining $\mathrm{AD}$-related genes that may affect $\mathrm{AD}$ related drugs may be partly discovered in the gene symbols searching. These novelly identified AD-related genes may be the potential candidates for further drug development of AD. These results demonstrated that our proposed data mining method may be an improved AD pharmacogenomics study.

3.3. SNP Information of AD-Related Genes. The SNP statuses for $99 \mathrm{AD}$-related genes are also provided in Table 3. This SNP status for each gene is calculated from the online NCBI 


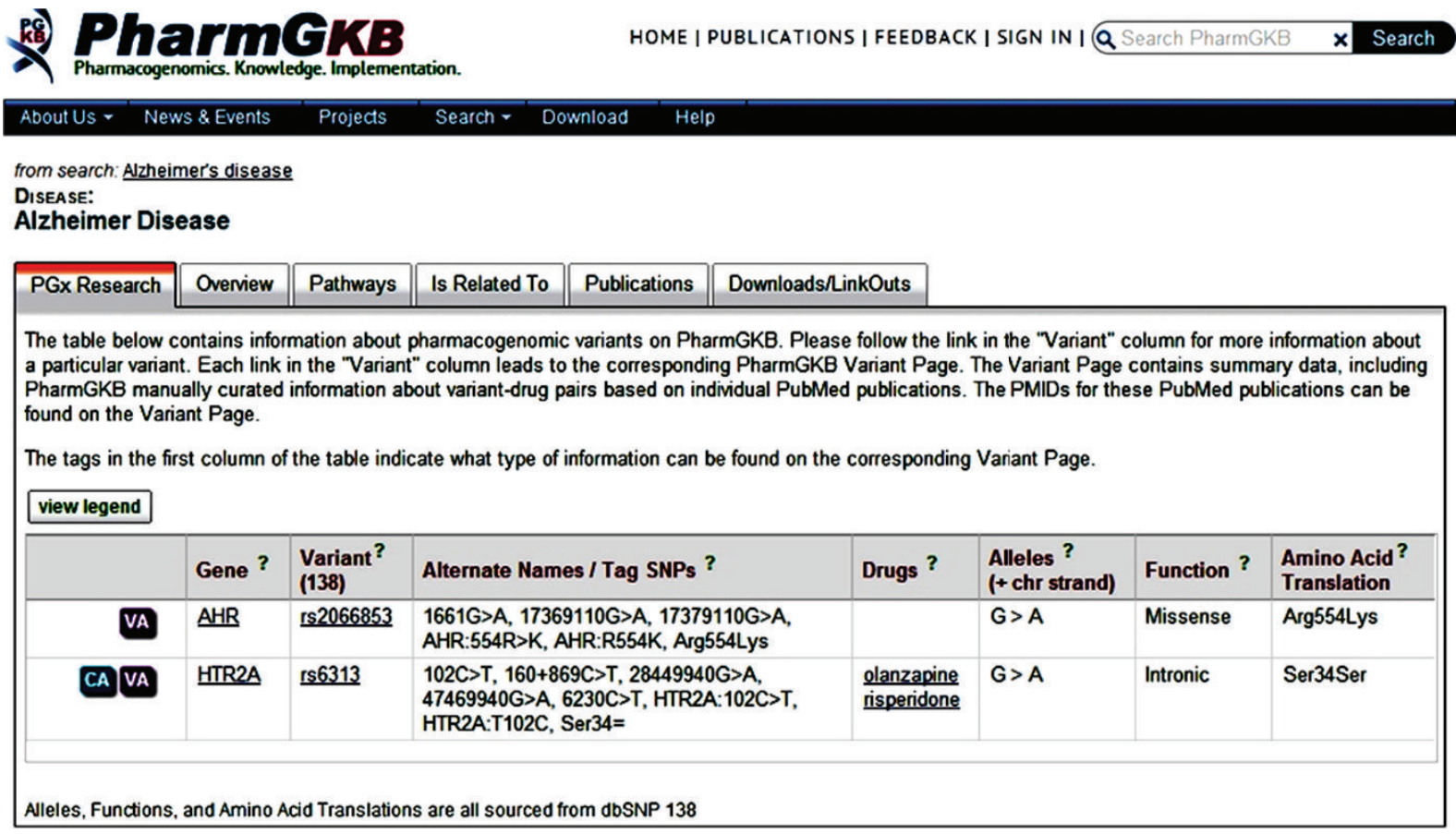

FIGURE 2: PharmGKB-pharmacogenomics online query for the variant information (SNP rs\#ID) of "Alzheimer's disease." Retrieval source: http://www.pharmgkb.org/disease/PA443319?previousQuery=Alzheimer's\%20disease.

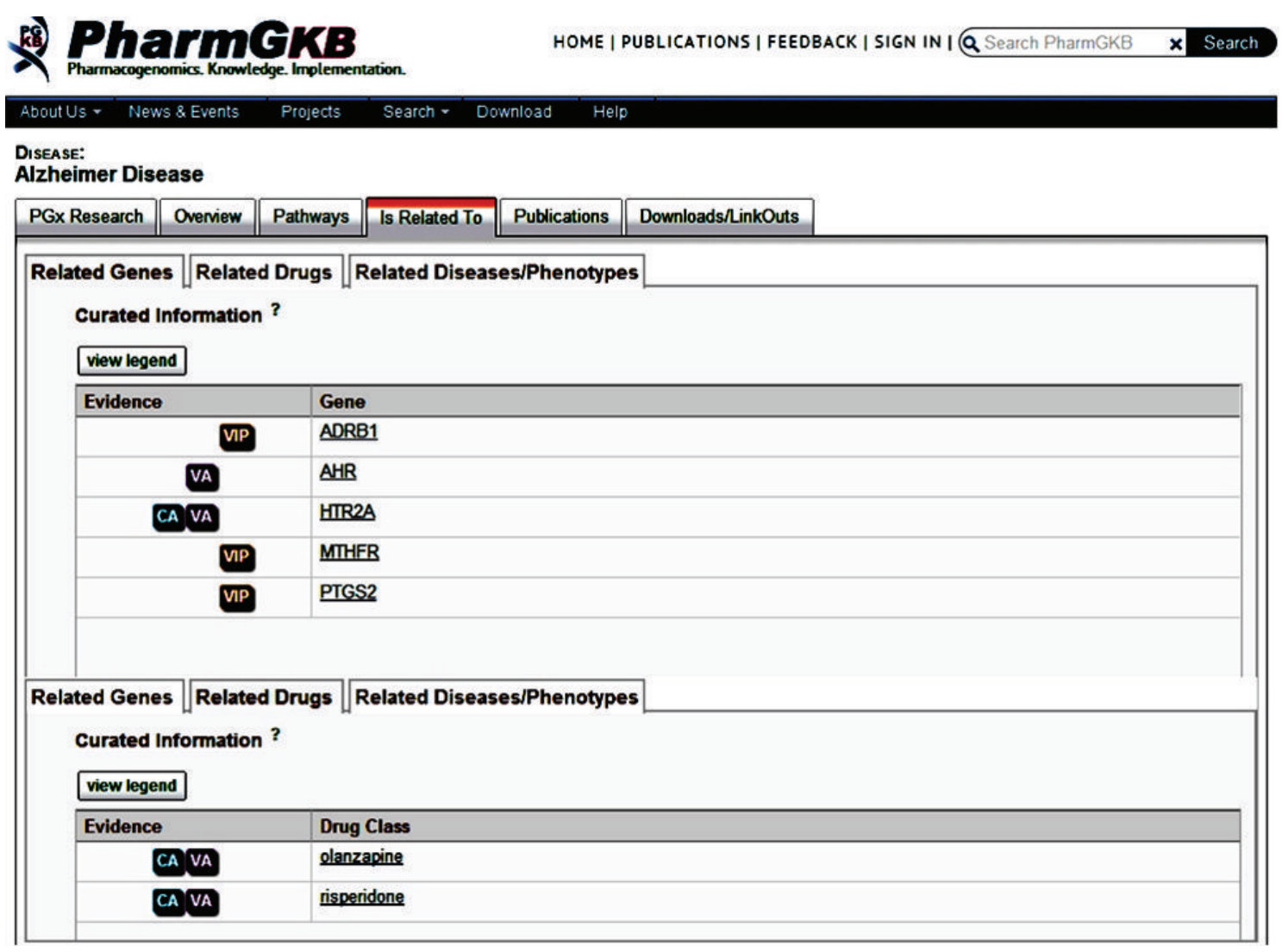

FIGURE 3: Gene and drug related information of "Alzheimer's disease" online query from PharmGKB. Retrieval source: http://www.pharmgkb .org/disease/PA443319?previousQuery=Alzheimer's\%20disease\#tabview=table 3\&subtab=33. 
TABLE 2: PharmGKB-based data mining results in terms of the PharmGKB accession ID, drug class, publications, and the number of gene information of Alzheimer's disease.

\begin{tabular}{|c|c|c|c|c|}
\hline No. & PharmGKB accession ID & Drug classes & Publications $^{* 1}$ & Gene no. $^{* 2}$ \\
\hline 1 & PA164712423 & Anticholinesterases & PMID: 206445622064456214674789 & 6 \\
\hline 2 & PA164712308 & Ace inhibitors, plain & PMID: 17362841 & 24 \\
\hline 3 & PA449515 & Etanercept & PMID: 19027875 & 12 \\
\hline 4 & PA451262 & Rivastigmine & $\begin{array}{l}\text { PMID: } 206445621632325317082448 \\
206445621528979717522596\end{array}$ & 2 \\
\hline 5 & PA450243 & Lithium & PMID: 17082448 & 13 \\
\hline 6 & PA10384 & $\begin{array}{l}\text { Anti-inflammatory and } \\
\text { antirheumatic products, } \\
\text { nonsteroids }\end{array}$ & PMID: 1708244817082448 & 11 \\
\hline 7 & PA449760 & Glatiramer acetate & PMID: 17082448 & 4 \\
\hline 8 & PA133950441 & Hmg coa reductase inhibitors & PMID: 17082448 & 39 \\
\hline 9 & PA151958596 & Curcumin & PMID: 17082448 & 2 \\
\hline 10 & PA451898 & Vitamin c & PMID: 17082448 & 16 \\
\hline 11 & PA451900 & Vitamin e & PMID: 17082448 & 1 \\
\hline 12 & PA452229 & Antidepressants & PMID: 17082448 & 43 \\
\hline 13 & PA452233 & Antipsychotics & PMID: 17082448 & 46 \\
\hline 14 & PA449726 & Galantamine & $\begin{array}{l}\text { PMID: } 206445621632325317082448 \\
15853556206445621467478912177686\end{array}$ & 7 \\
\hline 15 & PA10364 & Memantine & PMID: 17082448 & 0 \\
\hline 16 & PA451283 & Rosiglitazone & PMID: 16770341 & 34 \\
\hline 17 & PA448031 & Acetylcholine & PMID: 15695160 & 8 \\
\hline 18 & PA450626 & Nicotine & PMID: 15695160 & 88 \\
\hline 19 & PA137179528 & Nimesulide & PMID: 1633130311810182 & 3 \\
\hline 20 & PA449394 & Donepezil & $\begin{array}{l}\text { PMID: } 208592442064456216323253 \\
\text { l6424819 } 17082448206445621973817012142731\end{array}$ & 9 \\
\hline 21 & PA451576 & Tacrine & $\begin{array}{l}\text { PMID: } 95212541708244810801254 \\
977742718004213\end{array}$ & 6 \\
\hline 22 & PA448976 & Choline & PMID: 8618881 & 122 \\
\hline
\end{tabular}

${ }^{* 1}$ PMID: PubMed article ID number.

*2 The full gene names for each of the "drug classes" have been provided in the Supplementary file 1.

dbSNP queries. In general, many SNPs are found in these ADrelated genes. Some SNPs of these genes have been reported to be associated with AD. For example, the APOE gene is found in Table 3 and one of its SNPs, such as ApoE epsilon 4 allele, has been reported to be associated with AD [14]. With suitable tools for SNP genotyping, these SNP candidates are warranted for the pharmacogenomics research of AD.

Currently, there are many high throughput SNP genotyping methods developed (as shown in Figure 1), including PCR resequencing [15], TaqMan probes [16], SNP microarrays [17], Matrix Assisted Laser Desorption/Ionization-Time of Flight (MALDI-TOF) [18], and others [19, 20]. Furthermore, some SNP genotyping tools or databases are also developed, such as SNP-RFLPing2 for comprehensive PCR-RFLP information based on SNPs [21-24], algorithmic PCR-RFLP primer design and restriction enzymes for SNP genotyping $[25,26]$, and primer design for PCR-confronting two-pair primers (PCR-CTPP) [27, 28]. These tools and methods can provide useful and convenient information for SNP genotyping in the $\mathrm{AD}$ pharmacogenomics studies.

\section{Conclusions}

$\mathrm{AD}$ is the most common form of dementia for older people. The pharmacogenomics of $\mathrm{AD}$ still remains a challenge. In this study, we propose the pharmGKB-based data mining method to improve the gene discoveries for the potential AD-related drug candidates. With the assistance of bioinformatics, this improvement can help researchers to develop personal therapeutic drugs of AD.

\section{Conflict of Interests}

The authors declare that there is no conflict of interests regarding the publication of this paper. 







\section{Acknowledgments}

This work is partly supported by the National Science Council (NSC) in Taiwan under Grant nos. NSC1012622-E151-027-CC3, NSC101-2221-E-464-001, NSC101-2320B-037-049, NSC102-2221-E151-024-MY3, NSC102-2221-E214039, and NSC102-2221-E-464-004, by the National Sun YatSen University-KMU Joint Research Project (no. NSYSUKMU 103-p014), and by the Ministry of Health and Welfare, Taiwan (MOHW103-TD-B-111-05).

\section{References}

[1] N. C. Berchtold and C. W. Cotman, "Evolution in the conceptualization of dementia and Alzheimer's disease: Greco-Roman period to the 1960s," Neurobiology of Aging, vol. 19, no. 3, pp. 173-189, 1998.

[2] M.-Y. Shiau, L. Yu, H.-S. Yuan, J.-H. Lin, and C.-K. Liu, "Functional performance of Alzheimer's disease and vascular dementia in southern Taiwan," The Kaohsiung Journal of Medical Sciences, vol. 22, no. 9, pp. 437-446, 2006.

[3] W. Thies and L. Bleiler, "2013 Alzheimer's disease facts and figures," Alzheimer's \& Dementia, vol. 9, no. 2, pp. 208-245, 2013.

[4] L. Kruglyak and D. A. Nickerson, "Variation is the spice of life," Nature Genetics, vol. 27, no. 3, pp. 234-236, 2001.

[5] J. Voisey and C. P. Morris, "SNP technologies for drug discovery: a current review," Current Drug Discovery Technologies, vol. 5, no. 3, pp. 230-235, 2008.

[6] H. W. Chang, L. Y. Chuang, M. T. Tsai, and C. H. Yang, "The importance of integrating SNP and cheminformatics resources to pharmacogenomics," Current Drug Metabolism, vol. 13, no. 7, pp. 991-999, 2012.

[7] K. Sangkuhl, D. S. Berlin, R. B. Altman, and T. E. Klein, "PharmGKB: understanding the effects of individual genetic variants," Drug Metabolism Reviews, vol. 40, no. 4, pp. 539-551, 2008.

[8] K. M. Giacomini, C. M. Brett, R. B. Altman et al., "The pharmacogenetics research network: from SNP discovery to clinical drug response," Clinical Pharmacology \& Therapeutics, vol. 81, no. 3, pp. 328-345, 2007.

[9] T. E. Klein, J. T. Chang, M. K. Cho et al., "Integrating genotype and phenotype information: an overview of the PharmGKB project. Pharmacogenetics Research Network and Knowledge Base," The Pharmacogenomics Journal, vol. 1, no. 3, pp. 167-170, 2001.

[10] L. Gong, R. P. Owen, W. Gor, R. B. Altman, and T. E. Klein, "PharmGKB: an integrated resource of pharmacogenomic data and knowledge," Current Protocols in Bioinformatics, vol. 23, pp. 14.7.1-14.7.17, 2008.

[11] G. Navarro, "A guided tour to approximate string matching," ACM Computing Surveys, vol. 33, no. 1, pp. 31-88, 2001.

[12] M. Gilleland, "Levenshtein distance, in three flavors," Merriam Park Software, 2009, http://people.cs.pitt.edu/ kirk/cs1501/ Pruhs/Spring2006/assignments/editdistance/Levenshtein\% 20Distance.htm.

[13] R. Agrawal and R. Srikant, "Fast algorithms for mining association rules in large databases," in Proceedings of the 20th International Conference on Very Large Data Bases (VLDB '94), pp. 487-499, Santiago, Chile, 1994.
[14] Y. C. Yen, C. K. Liu, F. W. Lung, and M. Y. Chong, "Apolipoprotein E polymorphism and Alzheimer's disease," The Kaohsiung Journal of Medical Sciences, vol. 17, no. 4, pp. 190-197, 2001.

[15] J. Zhang, D. A. Wheeler, I. Yakub et al., "SNPdetector: a software tool for sensitive and accurate SNP detection," PLoS Computational Biology, vol. 1, no. 5, article e53, 2005.

[16] P. Borgiani, C. Ciccacci, V. Forte et al., "CYP4F2 genetic variant (rs2108622) significantly contributes to warfarin dosing variability in the Italian population," Pharmacogenomics, vol.10, no. 2, pp. 261-266, 2009.

[17] S. Sõber, E. Org, K. Kepp et al., “Targeting 160 candidate genes for blood pressure regulation with a genome-wide genotyping array," PLoS ONE, vol. 4, no. 6, Article ID e6034, 2009.

[18] T. J. Griffin and L. M. Smith, "Single-nucleotide polymorphism analysis by MALDI-TOF mass spectrometry," Trends in Biotechnology, vol. 18, no. 2, pp. 77-84, 2000.

[19] P.-Y. Kwok, "SNP genotyping with fluorescence polarization detection," Human Mutation, vol. 19, no. 4, pp. 315-323, 2002.

[20] M. Olivier, “The Invader assay for SNP genotyping," Mutation Research, vol. 573, no. 1-2, pp. 103-110, 2005.

[21] M. Ota, H. Fukushima, J. K. Kulski, and H. Inoko, "Single nucleotide polymorphism detection by polymerase chain reaction-restriction fragment length polymorphism," Nature protocols, vol. 2, no. 11, pp. 2857-2864, 2007.

[22] H.-W. Chang, C.-H. Yang, P.-L. Chang, Y.-H. Cheng, and L.-Y. Chuang, "SNP-RFLPing: restriction enzyme mining for SNPs in genomes," BMC Genomics, vol. 7, article 30, 2006.

[23] L.-Y. Chuang, C.-H. Yang, K.-H. Tsui et al., "Restriction enzyme mining for SNPs in genomes," Anticancer Research, vol. 28, no. 4, pp. 2001-2007, 2008.

[24] H.-W. Chang, Y.-H. Cheng, L.-Y. Chuang, and C.-H. Yang, "SNP-RFLPing 2: an updated and integrated PCR-RFLP tool for SNP genotyping," BMC Bioinformatics, vol. 11, article 173, 2010.

[25] C.-H. Yang, Y.-H. Cheng, C.-H. Yang, and L.-Y. Chuang, "Mutagenic primer design for mismatch PCR-RFLP SNP genotyping using a genetic algorithm," IEEE/ACM Transactions on Computational Biology and Bioinformatics, vol. 9, no. 3, pp. 837845, 2012.

[26] L. Y. Chuang, Y. H. Cheng, C. H. Yang, and C. H. Yang, "Associate PCR-RFLP assay design with SNPs based on genetic algorithm in appropriate parameters estimation," IEEE Transactions on NanoBioscience, vol. 12, no. 2, pp. 119-127, 2013.

[27] N. Hamajima, "PCR-CTPP: a new genotyping technique in the era of genetic epidemiology," Expert Review of Molecular Diagnostics, vol. 1, no. 1, pp. 119-123, 2001.

[28] C.-H. Yang, Y.-H. Cheng, L.-Y. Chuang, and H.-W. Chang, "Confronting two-pair primer design for enzyme-free SNP genotyping based on a genetic algorithm," BMC Bioinformatics, vol. 11, article 509, 2010. 

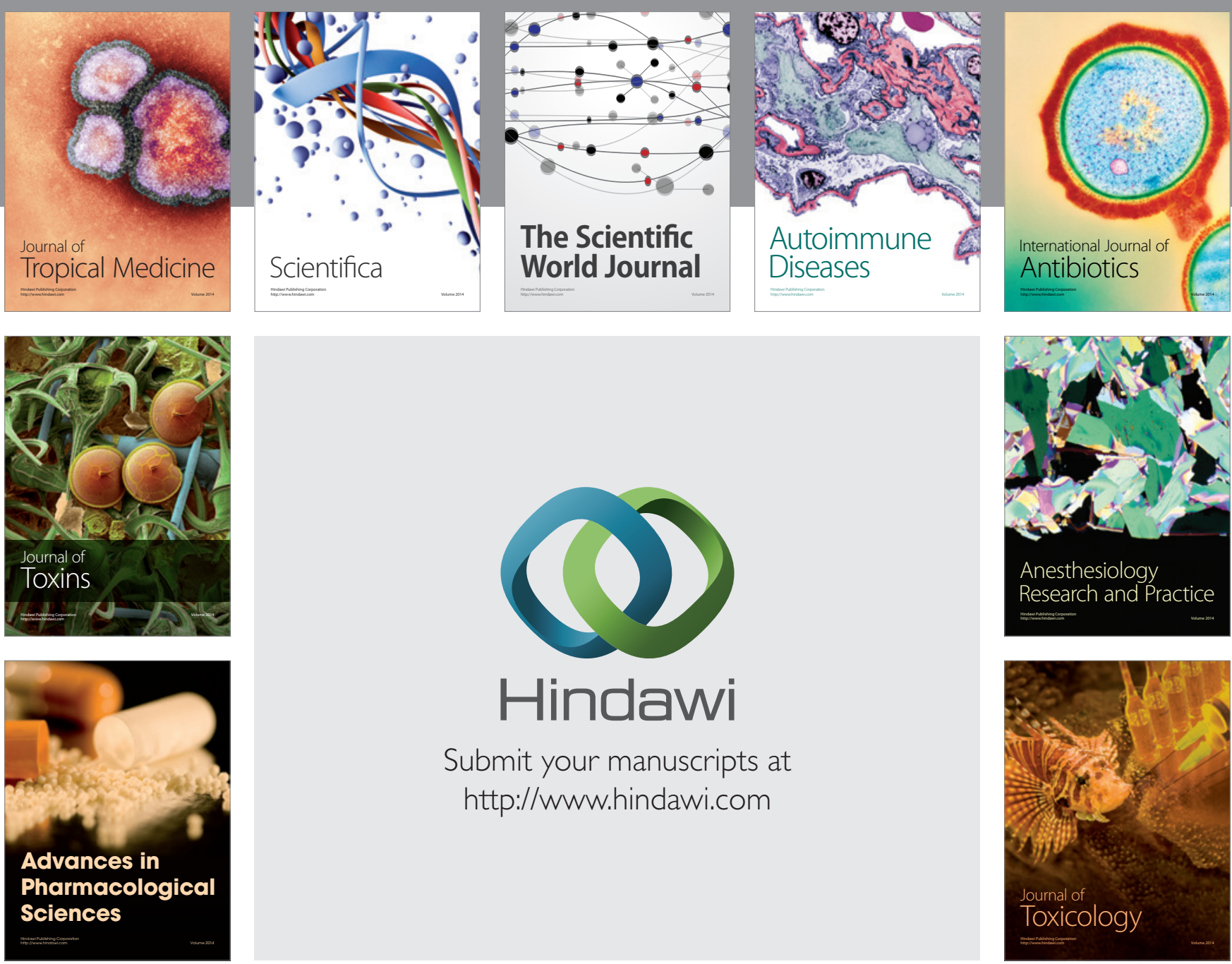

\section{Hindawi}

Submit your manuscripts at

http://www.hindawi.com
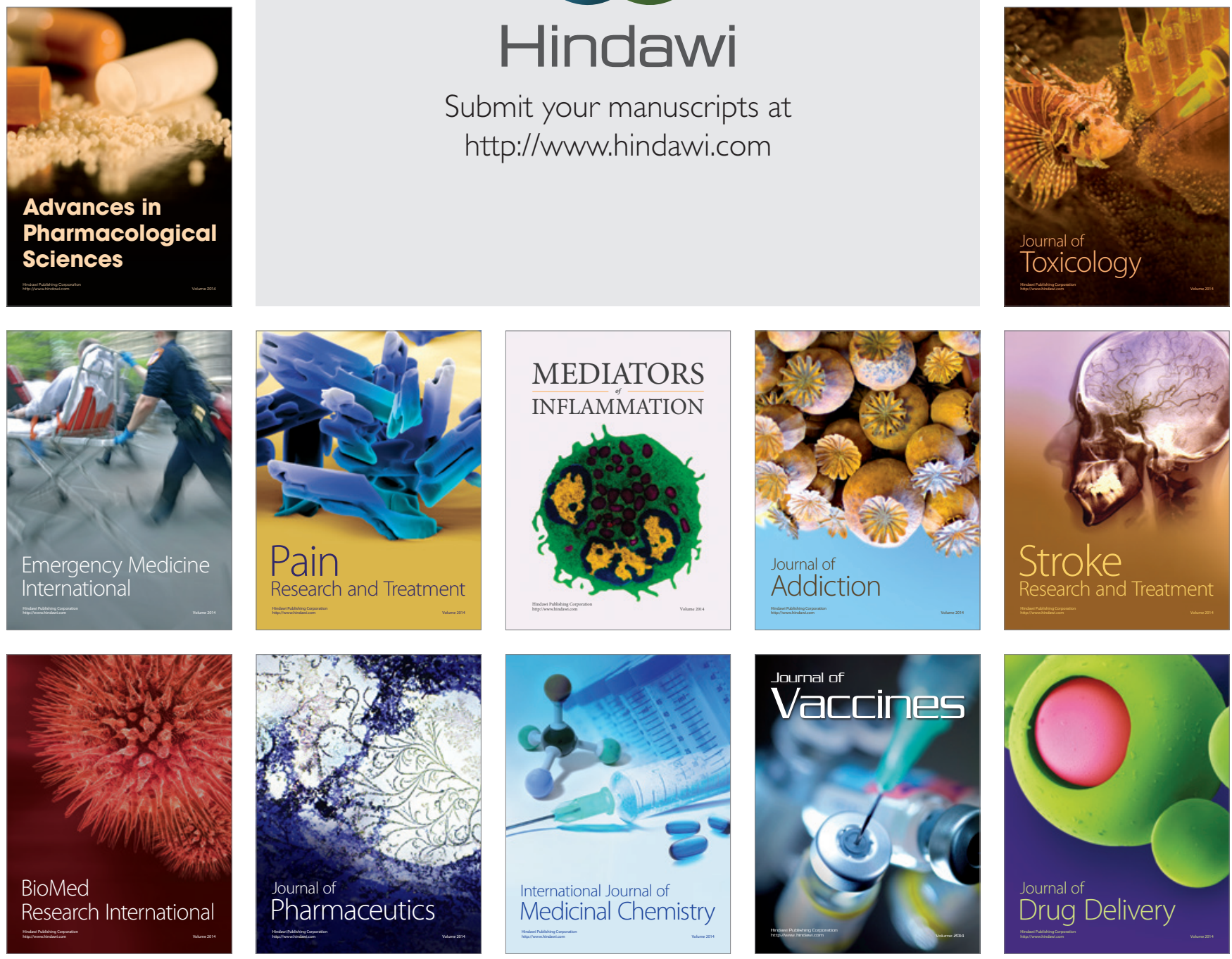